\title{
Restrained Step Domination Number for Some Amusing Product Graph of Paths and Cycle
}

\author{
G.Mahadevan, M.Vimala Suganthi
}

Abstract: G. Mahadevan, et, al., introduced the concept of restrained step domination number of a graph. A set $\mathrm{S} \subseteq \mathrm{V}$ of a graph $G$ is said to be restrained step dominating set, if $\langle S\rangle$ is the restrained dominating set and $\langle V-S\rangle$ is a perfect matching. The minimum cardinality taken over all the restrained step dominating set is called the restrained step domination number of $G$ and is denoted by $Y_{\mathrm{rgd}}(G)$. In this paper we explore this parameter for some product graph of path and cycle.

Keywords : complementary perfect domination, Restrained domination, restrained step domination.

\section{INTRODUCTION}

Paulraj Joseph et.al., [4], in the year 2006 introduced the concept of complementary perfect domination. A set is called a complementary perfect dominating set if $\mathrm{S}$ is a dominating set of $\mathrm{G}$ and the induced subgraph $\langle\mathrm{V}-\mathrm{S}\rangle$ has a perfect matching. The minimum cardinality taken over all complementary perfect dominating sets is called the complementary perfect domination number and is denoted by $\gamma_{\mathrm{cp}}(\mathrm{G})$. Further results of complementary perfect domination number is discussed in $[7,12]$. The concept of restrained domination number was introduced by Gayla.S et.al., in the year 1999 [1]. A dominating set is said to be restrained dominating set if every vertex in $\langle V-S\rangle$ is adjacent to atleast one vertex in $\mathrm{S}$ as well as in $\mathrm{V}-\mathrm{S}$. The minimum cardinality taken over all restrained dominating sets in $G$ is restrained dominating number and denoted by $\gamma_{\mathrm{r}}(\mathrm{G})$. Further results of restrained dominating number is been discussed in $[8,11]$. Inspired by the above, imposing a condition on the complement of restrained dominating set, G. Mahadevan, et.al., [5] introduced the concept of restrained step domination number of a graph in the year 2018. A set $\mathrm{S} \subseteq \mathrm{V}$ of a graph $\mathrm{G}$ is said to be restrained step dominating set, if $\langle S\rangle$ is the restrained dominating set and $\langle V-S\rangle$ is a perfect matching. The minimum cardinality taken over all the restrained step dominating set is called the restrained step dominating number of $G$ and is denoted by $\gamma_{\text {rad }}(G)$.

Revised Manuscript Received on December 5, 2019.

Dr.G.Mahadevan, Asst. Professor, Dept. of Mathematics, Gandhigram Rural Institute-Deemed to be University, Gandhigram.

M. Vimala Suganthi, Research Scholar, Dept. of Mathematics, Gandhigram Rural Insitute, Deemed to be University, Gandhigram.
The corona $\mathrm{G}_{1} \odot \mathrm{G}_{2}$ is defined as the graph $\mathrm{G}$ obtained by taking one copy of $\mathrm{G}_{1}$ of order $\mathrm{p}_{1}$ and $\mathrm{p}_{1}$ copies of $\mathrm{G}_{2}$ and then joining the $\mathrm{i}^{\mathrm{th}}$ vertex of $\mathrm{G}_{1}$ to every vertex in the $i^{\text {th }}$ copy of $G_{2}$. In this graph vertices are denoted as $v_{i}$ and $w_{j}^{i}$ where $v_{i}$ are the vertices of the graph $G_{1}, w_{j}$ are the vertices of the graph $G_{2}$ and $w_{j}{ }_{j}^{i}$ denotes the vertices of the copies of the graph $G_{2}$ attached to the vertex of $G_{1}$. For any two simple

graphs $\mathrm{G}$ and $\mathrm{H}$, the tensor product of $\mathrm{G}$ and $\mathrm{H}$ has vertex set $\mathrm{V}(\mathrm{G} \otimes \mathrm{H})=\mathrm{V}(\mathrm{G}) \times \mathrm{V}(\mathrm{H})$, edge set $\mathrm{E}(\mathrm{G} \otimes \mathrm{H})=\{(\mathrm{a}, \mathrm{b})(\mathrm{c}, \mathrm{d}) / \mathrm{ac}$ $\in \mathrm{E}(\mathrm{G})$ and $b d \in \mathrm{E}(\mathrm{H})\}$.

Preliminary result: We use the following preliminary result in our subsequent discussions.

Theorem 1.1 [5] For a connected graph $\mathrm{C}_{\mathrm{p}}, p \geq 3$, $\gamma_{\text {rsd }}\left(\mathrm{C}_{\mathrm{p}}\right)= \begin{cases}\frac{\mathrm{p}}{3} & \text { if } \mathrm{p} \equiv 0(\bmod 3) \\ \frac{\mathrm{p}+2}{3} & \text { if } \mathrm{p} \equiv 1(\bmod 3) \\ \frac{\mathrm{p}+4}{3} & \text { if } \mathrm{p} \equiv 2(\bmod 3) .\end{cases}$

Theorem 1.2 [13]If $n \leq p$ and $p \equiv 0,1(\bmod 3)$, then $\gamma_{\text {rate }}\left(\mathrm{C}_{\mathrm{p}} \otimes \mathrm{C}_{\mathrm{n}}\right)$

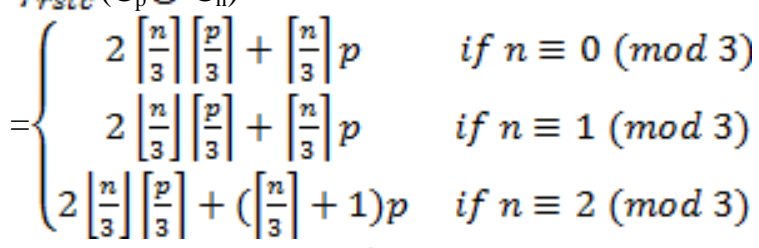

Theorem 1.3 [ 13] If $n \leq p$ and $p \equiv 2(\bmod 3)$, then $\gamma_{\text {rste }}\left(\mathrm{C}_{\mathrm{p}} \otimes \mathrm{C}_{\mathrm{n}}\right)$

$$
=\left\{\begin{aligned}
\left\lceil\frac{n}{3}\right\rceil\left(2\left\lceil\frac{p}{3}\right\rceil+p\right)+2 & \text { if } n & \equiv 0(\bmod 3) \\
\left\lfloor\frac{n}{3}\right\rfloor\left(2\left\lceil\frac{p}{3}\right\rceil\right)+2+\left\lceil\frac{n}{3}\right\rceil p & \text { if } n & \equiv 1(\bmod 3) \\
\left\lfloor\frac{n}{3}\right\rfloor\left(2\left\lceil\frac{p}{3}\right\rceil\right)+2+\left(\left\lceil\frac{n}{3}\right\rceil+1\right) p & \text { if } n & \equiv 2(\bmod 3)
\end{aligned}\right.
$$

\section{RESTRAINED STEP DOMINATION NUMBER OF CORONA PRODUCT OF GRAPHS}

Theorem 2.1 For a corona product $\mathrm{C}_{\mathrm{p}} \odot \mathrm{P}_{\mathrm{z}}$ where $\mathrm{C}_{\mathrm{p}}$ is the cycle with $p$ vertices and $P_{s}$ is the path with s vertices,

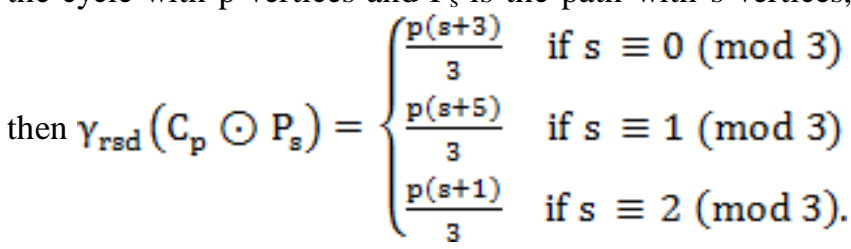
Proof Let $\mathrm{C}_{\mathrm{p}} \odot \mathrm{P}_{\mathrm{s}}$ be the corona product graph. Let the vertices in the cycle $C_{p}$ be $\left\{v_{1}, v_{2}, \ldots, v_{p}\right\}$ and the vertices in the path $\mathrm{P}_{\mathrm{s}}$ be $\left\{\mathrm{w}_{1}, \mathrm{w}_{2}, \ldots, \mathrm{w}_{\mathrm{s}}\right\}$ as $\mathrm{C}_{\mathrm{p}} \odot \mathrm{P}_{\mathrm{s}}$ is the corona product the vertices in the graph are 
$\left\{\mathrm{v}_{1}, \mathrm{v}_{2}, \ldots, \mathrm{v}_{\mathrm{p}}, \mathrm{w}_{1}{ }^{1}, \mathrm{w}_{2}{ }^{1}, \ldots, \mathrm{w}_{\mathrm{s}}{ }^{1}, \ldots, \mathrm{w}_{1}{ }^{\mathrm{p}}, \mathrm{w}_{2}{ }^{\mathrm{p}}, \ldots, \mathrm{w}_{\mathrm{s}}^{\mathrm{p}}\right\} . \quad$ Every vertices of the path $\mathrm{w}_{1}{ }^{\mathrm{i}}, \mathrm{w}_{2}{ }^{\mathrm{i}}, \ldots, \mathrm{w}_{\mathrm{s}}{ }_{\mathrm{i}}^{\mathrm{i}}$ is connected to the vertex $\mathrm{v}_{\mathrm{i}}$, where $1 \leq \mathrm{i} \leq \mathrm{p}$. In the graph $\mathrm{v}_{\mathrm{i}}, \mathrm{w}_{1}{ }^{\mathrm{i}}, \mathrm{w}_{2}, \ldots, \mathrm{w}_{\mathrm{s}}{ }^{\mathrm{i}}$, where $1 \leq \mathrm{i} \leq \mathrm{p}$ forms the cycle with $\mathrm{s}+1$ vetices. Hence $\gamma_{\text {rsd }}\left(\mathrm{C}_{\mathrm{p}} \odot \mathrm{P}_{\mathrm{s}}\right)=\mathrm{p}\left(\gamma_{\mathrm{rgd}}\left(\mathrm{C}_{\mathrm{s}+1}\right)\right)$, by theorem 1.1 implies

$$
\gamma_{\mathrm{rsd}}\left(\mathrm{C}_{\mathrm{p}} \odot \mathrm{P}_{\mathrm{s}}\right)= \begin{cases}\frac{p(s+3)}{3} & \text { if } s \equiv 0(\bmod 3) \\ \frac{p(s+5)}{3} & \text { if } s \equiv 1(\bmod 3) \\ \frac{p(s+1)}{3} & \text { if } s \equiv 2(\bmod 3) .\end{cases}
$$

Example Consider the graphs $\mathrm{C}_{3} \odot \mathrm{P}_{3}, \mathrm{C}_{3} \odot \mathrm{P}_{4}$, $\mathrm{C}_{3} \odot \mathrm{P}_{5}$

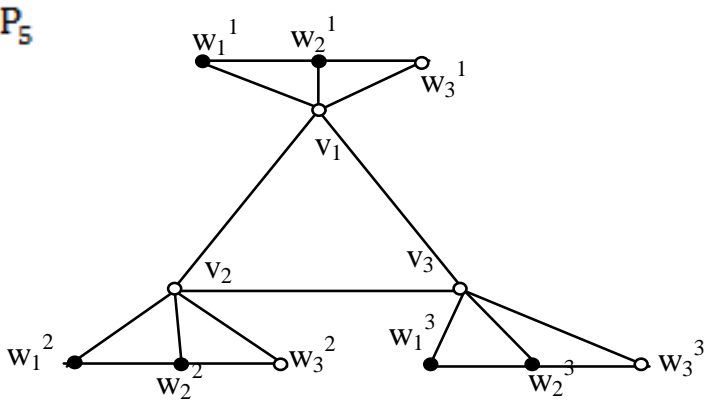

Figure 2.1

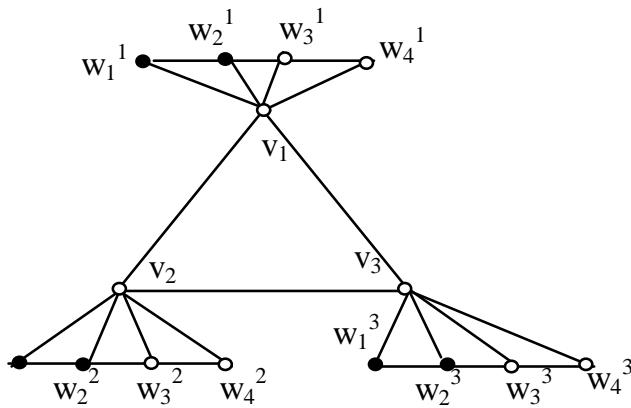

Figure 2.2

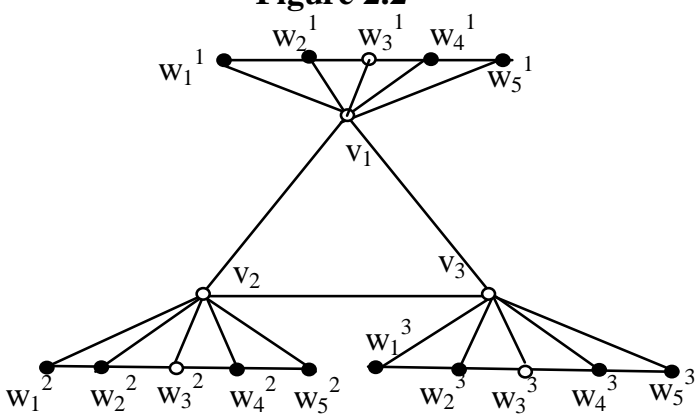

Figure 2.3

Illustration In figure 2.1, $\mathrm{S}=\left\{\mathrm{v}_{1}, \mathrm{v}_{2}, \mathrm{v}_{3}, \mathrm{w}_{3}{ }^{1}, \mathrm{w}_{3}{ }^{2}, \mathrm{w}_{3}{ }^{3}\right\}$ is the rsd-set, $|\mathrm{S}|=$ 6. $\quad \gamma_{\mathrm{rrd}}\left(\mathrm{C}_{\mathrm{p}} \odot \mathrm{P}_{\mathrm{s}}\right)=\frac{\mathrm{p}(\mathrm{s}+3)}{3}$, if $s \equiv 0(\bmod 3)$

Hence

$\gamma_{\text {rsd }}\left(\mathrm{C}_{3} \odot \mathrm{P}_{3}\right)=\frac{3(3+3)}{3}=6 . \quad$ In figure 2.2 , $\mathrm{S}=\left\{\mathrm{v}_{1}, \mathrm{v}_{2}, \mathrm{v}_{3}, \mathrm{w}_{3}{ }^{1}, \mathrm{w}_{4}{ }^{1}, \mathrm{w}_{3}{ }^{2}, \mathrm{w}_{4}{ }^{2}, \mathrm{w}_{3}{ }^{3}, \mathrm{w}_{4}{ }^{3}\right\}$ is the rsd-set, $|\mathrm{S}|=9$. $\gamma_{\mathrm{rad}}\left(\mathrm{C}_{\mathrm{p}} \odot \mathrm{P}_{\mathrm{s}}\right)=\frac{\mathrm{p}(\mathrm{s}+5)}{3}$, if $\mathrm{s} \equiv 1(\bmod 3)$. Hence $\gamma_{\mathrm{rgd}}\left(\mathrm{C}_{3} \odot \mathrm{P}_{4}\right)=\frac{3(4+5)}{3}=9 . \quad$ In figure 2.3 , $\mathrm{S}=\left\{\mathrm{v}_{1}, \mathrm{v}_{2}, \mathrm{v}_{3}, \mathrm{w}_{3}{ }^{1}, \mathrm{w}_{3}{ }^{2}, \mathrm{w}_{3}{ }^{3}\right\} \quad$ is the rsd-set, $|\mathrm{S}|=6$.
$\gamma_{\mathrm{rsd}}\left(\mathrm{C}_{\mathrm{p}} \odot \mathrm{P}_{\mathrm{s}}\right)=\frac{\mathrm{p}(\mathrm{s}+1)}{3}$, if $\mathrm{s} \equiv 0(\bmod 3)$. Hence $\gamma_{\mathrm{rgd}}\left(\mathrm{C}_{3} \odot \mathrm{P}_{5}\right)=\frac{3(5+1)}{3}=6$.

Theorem 2.2 For a corona product $P_{p} \odot P_{s}$ where $C_{p}$ and $P_{s}$ is the paths with $p$ and $s$ vertices, then $\gamma_{\mathrm{rgd}}\left(\mathrm{P}_{\mathrm{p}} \odot \mathrm{P}_{\mathrm{s}}\right)= \begin{cases}\frac{\mathrm{p}(\mathrm{s}+3)}{3} & \text { if } \mathrm{s} \equiv 0(\bmod 3) \\ \frac{\mathrm{p}(\mathrm{s}+5)}{3} & \text { if } \mathrm{s} \equiv 1(\bmod 3) \\ \frac{\mathrm{p}(\mathrm{s}+1)}{3} & \text { if } \mathrm{s} \equiv 2(\bmod 3) .\end{cases}$

Proof As $\gamma_{r s d}\left(P_{p} \odot P_{s}\right)=\gamma_{r s d}\left(C_{p} \odot P_{s}\right)$ the proof is same as the theorem 2.1

Theroem 2.3 For a corona product $P_{p} \odot C_{s}$ where $P_{p}$ is the path with $p$ vertices and $C_{s}$ is the cycle with $s$ vertices, then $\gamma_{\mathrm{rgd}}\left(\mathrm{P}_{\mathrm{p}} \odot \mathrm{C}_{\mathrm{s}}\right)= \begin{cases}\frac{\mathrm{p}(\mathrm{s}+3)}{3} & \text { if } \mathrm{s} \equiv 0(\bmod 3) \\ \frac{\mathrm{p}(\mathrm{s}+5)}{3} & \text { if } \mathrm{s} \equiv 1(\bmod 3) \\ \frac{\mathrm{p}(\mathrm{s}+7)}{3} & \text { if } \mathrm{s} \equiv 2(\bmod 3) .\end{cases}$

Proof Let $\mathrm{P}_{\mathrm{p}} \odot \mathrm{C}_{\mathrm{s}}$ be the corona product graph. Let the vertices in the path $P_{p}$ be $\left\{w_{1}, w_{2}, \ldots, w_{p}\right\}$ and let the vertices in the cycle $C_{s}$ be $\left\{v_{1}, v_{2}, \ldots, v_{s}\right\}$. Let the vertices in the product graph be $\left\{\mathrm{w}_{1}, \mathrm{w}_{2}, \ldots, \mathrm{w}_{\mathrm{p}}, \quad \mathrm{v}_{1}{ }^{1}, \mathrm{v}_{2}{ }^{1}, \ldots \mathrm{v}_{\mathrm{s}}{ }^{1}, \ldots, \mathrm{v}_{1}{ }^{\mathrm{p}}, \mathrm{v}_{2}{ }^{\mathrm{p}}, \ldots, \mathrm{v}_{\mathrm{s}}{ }^{\mathrm{p}}\right\}$. Clearly, each vertices $\mathrm{w}_{\mathrm{i}}$ dominates the each cycles $\mathrm{v}_{1}{ }^{\mathrm{i}}, \mathrm{v}_{2}{ }^{\mathrm{i}}, \ldots \mathrm{v}_{\mathrm{s}}{ }^{\mathrm{i}}$ where, $1 \leq \mathrm{i} \leq \mathrm{p}$. Hence $\left\{\mathrm{w}_{1}, \mathrm{w}_{2}, \ldots, \mathrm{w}_{\mathrm{p}}\right\}$ is a rsd-set whose cardinality is $\mathrm{p}$. Also rsd-number for p-copies of the cycle $\mathrm{v}_{1}{ }_{1}^{\mathrm{i}}, \mathrm{v}_{2}{ }^{\mathrm{i}}, \ldots \mathrm{v}_{\mathrm{s}}{ }^{\mathrm{i}}$ where, $1 \leq \mathrm{i} \leq \mathrm{p}$ is $\mathrm{p} \cdot \gamma_{\text {rgd }}\left(\mathrm{C}_{\mathrm{g}}\right)$. Hence $\gamma_{\mathrm{rgd}}\left(\mathrm{P}_{\mathrm{p}} \odot \mathrm{C}_{\mathrm{s}}\right)=\mathrm{p}+\mathrm{p} \cdot \gamma_{\mathrm{rgd}}\left(\mathrm{C}_{\mathrm{s}}\right)$, by theorem 1.1.Therefore,

$\gamma_{\mathrm{rsd}}\left(\mathrm{P}_{\mathrm{p}} \odot \mathrm{C}_{\mathrm{s}}\right)= \begin{cases}\frac{\mathrm{p}(\mathrm{s}+3)}{3} & \text { if } \mathrm{s} \equiv 0(\bmod 3) \\ \frac{\mathrm{p}(\mathrm{s}+5)}{3} & \text { if } s \equiv 1(\bmod 3) \\ \frac{\mathrm{p}(\mathrm{s}+7)}{3} & \text { if } s \equiv 2(\bmod 3) .\end{cases}$

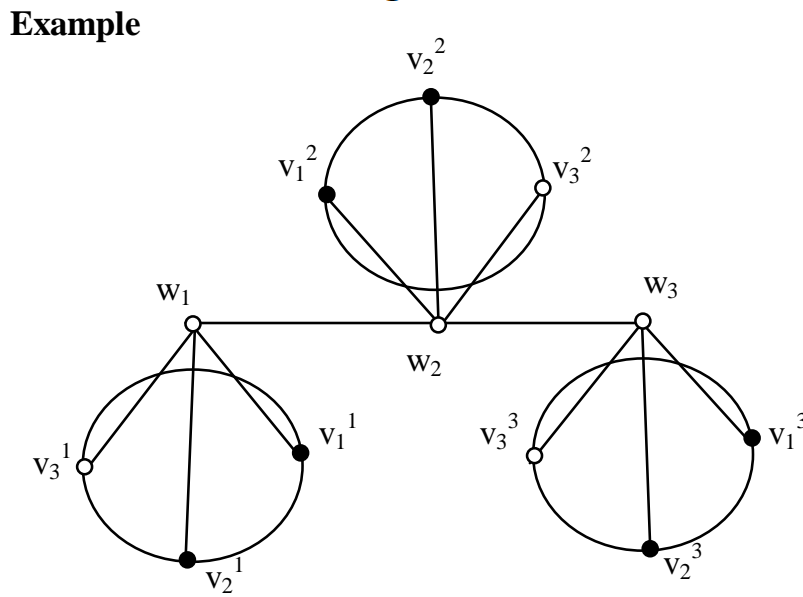

Figure 2.4 


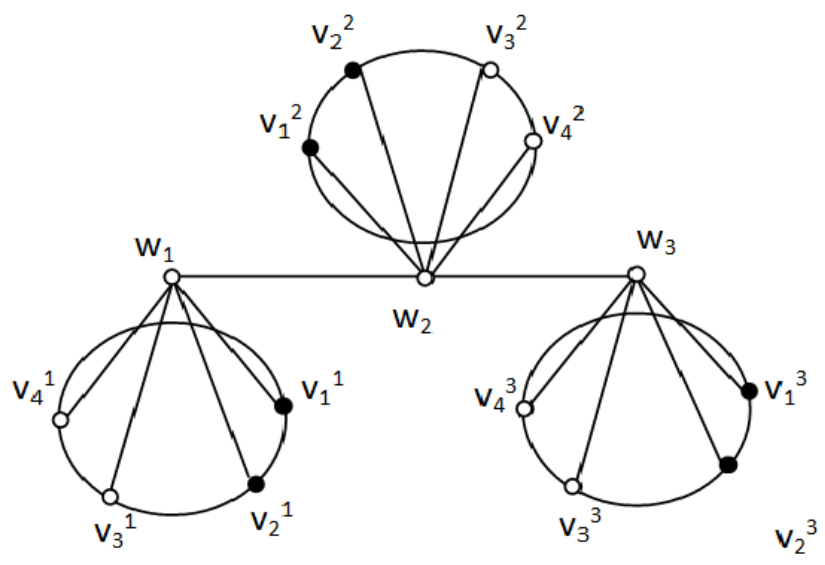

Figure 2.5

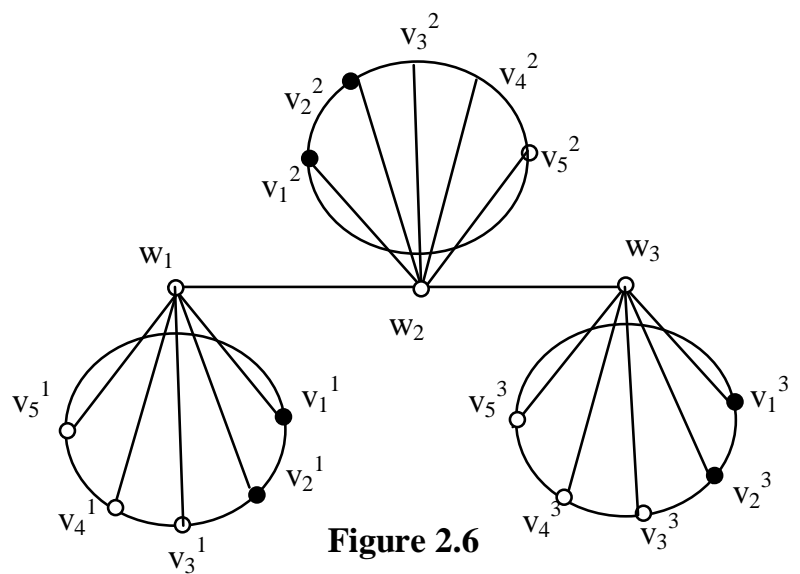

Illustration In figure 2.4, $\mathrm{S}=\left\{\mathrm{w}_{1}, \mathrm{w}_{2}, \mathrm{w}_{3}, \mathrm{v}_{3}{ }^{1}, \mathrm{v}_{3}{ }^{2}, \mathrm{v}_{3}{ }^{3}\right\}$ is the rsd-set, $\quad|\mathrm{S}|=$ 6. $\quad \gamma_{\mathrm{rsd}}\left(\mathrm{P}_{\mathrm{p}} \odot \mathrm{C}_{\mathrm{s}}\right)=\frac{\mathrm{p}(\mathrm{s}+3)}{3}$, if $\mathrm{s} \equiv 0(\bmod 3)$

$\gamma_{\mathrm{rgd}}\left(\mathrm{P}_{3} \odot \mathrm{C}_{3}\right)=\frac{3(3+3)}{3}=6 . \quad$ In figure 2.5 , $\mathrm{S}=\left\{\mathrm{w}_{1}, \mathrm{w}_{2}, \mathrm{w}_{3}, \mathrm{v}_{3}{ }^{1}, \mathrm{v}_{4}{ }^{1}, \mathrm{v}_{3}{ }^{2}, \mathrm{v}_{4}{ }^{2}, \mathrm{v}_{3}{ }^{3}, \mathrm{v}_{4}{ }^{3}\right\}$ is the rsd-set, $|\mathrm{S}|=9$. $\gamma_{\mathrm{rsd}}\left(\mathrm{P}_{\mathrm{p}} \odot \mathrm{C}_{\mathrm{s}}\right)=\frac{\mathrm{p}(\mathrm{s}+5)}{3}$, if $\mathrm{s} \equiv 1(\bmod 3)$. Hence $\gamma_{\mathrm{rgd}}\left(\mathrm{P}_{3} \odot \mathrm{C}_{4}\right)=\frac{3(4+5)}{3}=9 . \quad$ In figure 2.6, $\mathrm{S}=\{$ $\mathrm{w}_{1}, \mathrm{w}_{2}, \mathrm{w}_{3}, \mathrm{v}_{3}{ }^{1}, \mathrm{v}_{4}{ }^{1}, \mathrm{v}_{5}{ }^{1}, \mathrm{v}_{3}{ }^{2}, \mathrm{v}_{4}{ }^{2}, \mathrm{v}_{5}{ }^{2}, \mathrm{v}_{3}{ }^{3}, \mathrm{v}_{4}{ }^{3}, \mathrm{v}_{5}{ }^{3}$ \} is the rsd-set, $|\mathrm{S}|=12 \gamma_{\mathrm{rsd}}\left(\mathrm{P}_{\mathrm{p}} \odot \mathrm{C}_{\mathrm{s}}\right)=\frac{\mathrm{p}(\mathrm{s}+7)}{3}$, if $\mathrm{s} \equiv 2(\bmod 3)$. Hence $\gamma_{\mathrm{rgd}}\left(\mathrm{P}_{3} \odot \mathrm{C}_{5}\right)=\frac{3(5+7)}{3}=12$.

Theorem 2.4 For a corona product $\mathrm{C}_{\mathrm{p}} \odot \mathrm{C}_{\mathrm{s}}$ where $\mathrm{C}_{\mathrm{p}}$ and $\mathrm{C}_{\mathrm{s}}$ are the cycles with $\mathrm{r}$ and $\mathrm{s}$ vertices respectively, then $\gamma_{\mathrm{rgd}}\left(\mathrm{C}_{\mathrm{p}} \odot \mathrm{C}_{\mathrm{s}}\right)= \begin{cases}\frac{\mathrm{p}(\mathrm{s}+3)}{3} & \text { if } \mathrm{s} \equiv 0(\bmod 3) \\ \frac{\mathrm{p}(\mathrm{s}+5)}{3} & \text { if } \mathrm{s} \equiv 1(\bmod 3) \\ \frac{p(s+7)}{3} & \text { if } \mathrm{s} \equiv 2(\bmod 3) .\end{cases}$

Proof As $\gamma_{\mathrm{rsd}}\left(\mathrm{C}_{\mathrm{p}} \odot \mathrm{C}_{\mathrm{s}}\right)=\gamma_{\mathrm{rgd}}\left(\mathrm{P}_{\mathrm{p}} \odot \mathrm{C}_{\mathrm{s}}\right)$ the proof is same as the theorem 2.3.

\section{RESTRAINED STEP DOMINATION NUMBER FOR TENSOR PRODUCT OF GRAPHS}

Theorem 3.1 If $n \leq p$ and $p \equiv 0,1,2(\bmod 3)$ and $p$ is odd, then

$\gamma_{\text {rsd }}\left(\mathrm{P}_{\mathrm{n}} \otimes \mathrm{P}_{\mathrm{p}}\right)=$

$\left\{\begin{aligned} 2\left\lceil\frac{n}{3}\right\rceil\left[\frac{p}{3}\right\rceil+\left\lceil\frac{p}{3}\right\rceil n & \text { if } n & \equiv 0(\bmod 3) \\ 2\left\lceil\frac{n}{3}\right\rceil\left[\frac{p}{3}\right\rceil+\left\lceil\frac{p}{3}\right\rceil n & \text { if } n & \equiv 1(\bmod 3) \\ 2\left(\left\lceil\frac{n}{3}\right\rceil-1\right)\left\lceil\frac{p}{3}\right\rceil+\left\lceil\frac{p}{3}\right\rceil n & \text { if } n & \equiv 2(\bmod 3) .\end{aligned}\right.$

Proof: Let $\mathrm{P}_{\mathrm{n}} \otimes \mathrm{P}_{\mathrm{p}}$ be the tensor product graphs of two paths. Case 1: $n$ is odd. Let $S_{1}=\left\{v_{1 j}: j \equiv 0,1 \bmod 6\right\}, S_{2}=\left\{v_{2 j}\right.$ : $\mathrm{j} \equiv 2,4 \bmod 6\}, \mathrm{S}_{3}=\left\{\mathrm{v}_{\mathrm{ij}}: 3 \leq j \leq p, 1 \leq i \leq n-2\right.$ $\mathrm{i} \quad \equiv 2,4 \bmod 6$ and $j \equiv 1 \bmod 3\}, \quad \mathrm{S}_{4}=\left\{\mathrm{v}_{\mathrm{ij}}\right.$ : $3 \leq j \leq p \quad, \quad 1 \quad \leq i \leq n-2$ $\mathrm{i} \equiv 0,1 \bmod 6$ and $j \equiv 2 \bmod 3\} \quad, \quad \mathrm{S}_{5}=\left\{\mathrm{v}_{\mathrm{ij}}\right.$ : $3 \leq j \leq p, \quad 1 \leq i \leq n-2$ and $j \equiv 0 \bmod 3\}$ , $\mathrm{S}_{6}=\left\{\mathrm{v}_{\mathrm{n}-1\} \mathrm{j}}: 4 \leq j \leq p\right.$ andj $\left.\equiv 0 \bmod 3\right\} \cup\left\{\mathrm{v}_{\{\mathrm{n}-1\} 1}\right\}$ and $\mathrm{S}_{7}=\left\{\mathrm{v}_{\mathrm{ip}}: 1 \leq i \leq n\right\}$. If $n \equiv 0(\bmod 3), \quad \mathrm{S}=$ $\mathrm{S}_{1} \cup_{\mathrm{S}_{2}} \cup_{\mathrm{S}_{3}} \cup_{\mathrm{S}_{4}} \cup_{\mathrm{S}_{5}} \cup_{\mathrm{S}_{6}}$ is the restrained step dominating set whose cardinality is $2\left\lceil\frac{n}{3}\right\rceil\left[\frac{p}{3}\right\rceil+\left\lceil\frac{p}{3}\right\rceil n$. If $n \equiv 1(\bmod 3), \mathrm{S}=\mathrm{S}_{1} \cup \mathrm{S}_{2} \cup \mathrm{S}_{3} \cup \mathrm{S}_{4} \cup \mathrm{S}_{5} \cup \mathrm{S}_{6}$ is the restrained step dominating set whose cardinality is $2\left\lfloor\frac{n}{3}\right]\left\lceil\frac{p}{3}\right\rceil+\left[\frac{p}{3}\right] n . \quad$ If $\quad n \equiv 2(\bmod 3) \quad, \quad \mathrm{S}=$ $\mathrm{S}_{1} \cup_{S_{2}} \cup_{S_{3}} \cup_{S_{4}} \cup_{S_{5}} \cup_{S_{6}} \cup_{S_{7}}$ is the restrained step dominating set whose cardinality is $2\left(\left\lceil\frac{n}{3}\right\rceil-1\right)\left\lceil\frac{p}{3}\right\rceil+\left\lceil\frac{p}{3}\right\rceil n$.

Thus, $\gamma_{\text {rsd }}\left(\mathrm{C}_{\mathrm{n}} \otimes \mathrm{C}_{\mathrm{p}}\right) \leq|\mathrm{S}|$.

Case 2: $n$ is even . Let $S_{1}=\left\{v_{1 j}\right.$; $\left.\equiv 0,1 \bmod 6\right\}, S_{2}=\left\{v_{2 j}\right.$ : $\mathrm{j} \equiv 2,4 \bmod 6\}, \mathrm{S}_{3}=\left\{\mathrm{v}_{\mathrm{ij}}: 3 \leq j \leq p, 1 \leq i \leq n-2\right.$ $\mathrm{i} \quad \equiv 2,4 \bmod 6$ and $j \equiv 1 \bmod 3\}, \quad \mathrm{S}_{4}=\left\{\mathrm{v}_{\mathrm{ij}}\right.$ : $3 \leq j \leq p \quad 1 \quad 1 \quad \leq i \leq n$ i $\equiv 0,1 \bmod 6$ and $j \equiv 2 \bmod 3\} \quad, \quad \mathrm{S}_{5}=\left\{v_{\mathrm{ij}}\right.$ : $3 \leq j \leq p, \quad 1 \leq i \leq n$ and $j \equiv 0 \bmod 3\} \quad$ and $\mathrm{S}_{6}=\left\{\mathrm{v}_{\mathrm{ip}}: 1 \leq i \leq n\right\}$. If $n \equiv 0(\bmod 3), \mathrm{S}=$ $\mathrm{S}_{1} \cup \mathrm{S}_{2} \cup_{S_{3}} \cup_{S_{4}} \cup_{S_{5}}$ is the restrained step dominating set whose cardinality is $2\left\lceil\frac{n}{3}\right\rceil\left[\frac{p}{3}\right\rceil+\left\lceil\frac{p}{3}\right] n$. If $n \equiv 1(\bmod 3), \mathrm{S}=\mathrm{S}_{1} \cup_{\mathrm{S}_{2}} \cup_{\mathrm{S}_{3}} \cup_{\mathrm{S}_{4}} \cup_{\mathrm{S}_{5}}$ is the restrained step dominating set whose cardinality is $2\left\lfloor\frac{n}{3}\right]\left[\frac{p}{3}\right]+\left[\frac{p}{3} \mid n\right.$. If $n \equiv 2(\bmod 3), \mathrm{S}=\mathrm{S}_{1} \cup_{\mathrm{S}_{2}} \cup_{\mathrm{S}_{3}} \cup_{\mathrm{S}_{4}} \cup_{\mathrm{S}_{5}} \cup_{\mathrm{S}_{6}}$ is the restrained step dominating set whose cardinality is $2\left(\left\lceil\frac{n}{3}\right\rceil-1\right)\left\lceil\frac{p}{3}\right\rceil+\left\lceil\frac{p}{3}\right\rceil n$.

Thus, $\gamma_{r s d}\left(\mathrm{C}_{\mathrm{n}} \otimes \mathrm{C}_{\mathrm{p}}\right) \leq|\mathrm{S}|$.

In all the above cases $\gamma_{r s d} \leq|\mathrm{S}|$, if there exists a restrained step dominating set $\mathrm{T} \subseteq \mathrm{S}$, then the set $\langle\mathrm{V}-\mathrm{T}\rangle$ has atleast one non-independent $\mathrm{K}_{2}$, which contradicts the definition implies $\gamma_{\text {rsd }} \geq|\mathrm{S}|$. Hence $\gamma_{\text {rsd }}\left(\mathrm{C}_{\mathrm{n}} \otimes \mathrm{C}_{\mathrm{p}}\right)=|\mathrm{S}|$. 
Theorem 3.2 If $\mathrm{n} \leq \mathrm{p}$ and $\mathrm{p} \equiv 0,1,2(\bmod 3)$ and $\mathrm{p}$ is even, then

$$
\begin{aligned}
& \gamma_{\text {rsd }}\left(\mathrm{P}_{\mathrm{n}} \otimes \mathrm{P}_{\mathrm{p}}\right)= \\
& \left\{\begin{aligned}
2\left\lceil\frac{n}{3}\right\rceil\left[\frac{p}{3}\right\rceil+\left\lceil\frac{p}{3}\right\rceil n & \text { if } n & \equiv 0(\bmod 3) \\
2\left\lceil\frac{n}{3}\right\rfloor\left[\frac{p}{3}\right\rfloor+\left\lceil\frac{p}{3}\right\rceil n & \text { if } n & \equiv 1(\bmod 3) \\
2\left(\left\lceil\frac{n}{3}\right\rceil-1\right)\left\lceil\frac{p}{3}\right\rceil+\left\lfloor\frac{p}{3}\right\rfloor n & \text { if } n & \equiv 2(\bmod 3) .
\end{aligned}\right.
\end{aligned}
$$

Proof: Restrained step dominating sets are taken as in theorem 3.1, but differs only in cardinality i.e, Case $1: \mathrm{n}$ is odd. If $n \equiv 0$ ( $\bmod 3), S=S_{1} \cup_{S_{2}} \cup_{S_{3}} \cup_{S_{4}} \cup_{S_{5}} \cup_{S_{6}}$ is the restrained step dominating set whose cardinality is $2\left\lceil\frac{n}{3}\right\rceil\left\lceil\frac{p}{3}\right\rceil+\left\lceil\frac{p}{3}\right\rceil n . \quad$ If $\quad n \equiv 1(\bmod 3) \quad, \quad \mathrm{S}=$ $\mathrm{S}_{1} \cup_{S_{2}} \cup_{S_{3}} \cup_{S_{4}} \cup_{S_{5}} \cup_{S_{6}}$ is the restrained step dominating set whose cardinality is $2\left\lfloor\frac{n}{3}\right]\left\lceil\left[\frac{p}{3}\right\rceil+\left\lceil\frac{p}{3}\right\rceil n\right.$. If $n \equiv 2(\bmod 3), \mathrm{S}=\mathrm{S}_{1} \cup \mathrm{S}_{2} \cup \mathrm{S}_{3} \cup \mathrm{S}_{4} \cup \mathrm{S}_{5} \cup \mathrm{S}_{6} \cup \mathrm{S}_{7}$ is the restrained step dominating set whose cardinality is $2\left(\left\lceil\frac{n}{3}\right\rceil-1\right)\left\lceil\frac{p}{3}\right\rceil+\left\lceil\frac{p}{3}\right\rceil n$.

Thus, $\gamma_{\text {rsd }}\left(\mathrm{C}_{\mathrm{n}} \otimes \mathrm{C}_{\mathrm{p}}\right) \leq|\mathrm{S}|$.

Case $2: \mathrm{n}$ is even . If $n \equiv 0(\bmod 3), \mathrm{S}=$ $\mathrm{S}_{1} \cup \mathrm{S}_{2} \cup \mathrm{S}_{3} \cup \mathrm{S}_{4} \cup \mathrm{S}_{5}$ is the restrained step dominating set whose cardinality is $2\left\lceil\left\lceil\frac{n}{3}\right\rceil\left\lceil\frac{p}{3}\right\rceil+\left\lceil\frac{p}{3}\right\rceil n\right.$. If $n \equiv 1(\bmod 3), \mathrm{S}=\mathrm{S}_{1} \cup \mathrm{S}_{2} \cup_{S_{3}} \cup_{\mathrm{S}_{4}} \cup_{\mathrm{S}_{5}}$ is the restrained step dominating set whose cardinality is $2\left\lfloor\frac{n}{3}\right\rfloor\left[\frac{p}{3}\right\rceil+\left\lceil\frac{p}{3}\right\rceil n$. If $n \equiv 2(\bmod 3), \mathrm{S}=\mathrm{S}_{1} \cup \mathrm{S}_{2} \cup \mathrm{S}_{3} \cup \mathrm{S}_{4} \cup \mathrm{S}_{5} \cup \mathrm{S}_{6}$ is the restrained step dominating set whose cardinality is $2\left(\left\lceil\frac{n}{3}\right\rceil-1\right)\left\lceil\frac{p}{3}\right\rceil+\left\lceil\frac{p}{3}\right\rceil n$.

Thus, $\gamma_{\text {rsd }}\left(\mathrm{C}_{\mathrm{n}} \otimes \mathrm{C}_{\mathrm{p}}\right) \leq|\mathrm{S}|$.

In all the above cases $\gamma_{\text {rsd }} \leq|\mathrm{S}|$, if there exists a restrained step dominating set $\mathrm{T} \subseteq \mathrm{S}$, then the set $\langle\mathrm{V}-\mathrm{T}\rangle$ has atleast one non-independent $\mathrm{K}_{2}$, which contradicts the definition implies $\gamma_{\text {rsd }} \geq|\mathrm{S}|$. Hence $\gamma_{\text {rsd }}\left(\mathrm{C}_{\mathrm{n}} \otimes \mathrm{C}_{\mathrm{p}}\right)=|\mathrm{S}|$.

Example 3.1 consider the graph $\mathrm{P}_{6} \otimes \mathrm{P}_{6}$

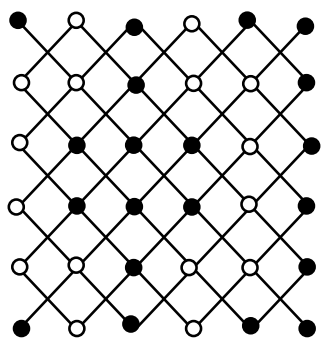

Figure 3.1

Here the darkened vertices are the restrained step dominating set . Whose cardinality is 20 .

$$
\begin{aligned}
& \gamma_{r s d}\left(\mathrm{P}_{\mathrm{p}} \otimes \mathrm{P}_{\mathrm{n}}\right)=2\left\lceil\frac{n}{3}\right\rceil\left\lceil\frac{p}{3}\right\rceil+\left\lceil\frac{n}{3}\right\rceil p \\
& \text { Implies } \gamma_{\text {rsd }}\left(\mathrm{P}_{6} \otimes \mathrm{P}_{6}\right)=2\left\lceil\frac{6}{3}\right\rceil\left[\frac{6}{3}\right\rceil+\left\lceil\frac{6}{3}\right\rceil 6=20
\end{aligned}
$$

Theorem 3.3 If $n \leq p$ and $p \equiv 0,1(\bmod 3)$, then $\gamma_{\text {rsd }}\left(\mathrm{C}_{\mathrm{p}} \otimes \mathrm{C}_{\mathrm{n}}\right)=$

$$
\left\{\begin{aligned}
2\left\lceil\left[\frac{n}{3}\right\rceil\left[\frac{p}{3}\right\rceil+\left\lceil\frac{n}{3}\right\rceil p\right. & \text { if } n & \equiv 0(\bmod 3) \\
2\left\lfloor\frac{n}{3}\right\rfloor\left[\frac{p}{3}\right\rceil+\left\lceil\frac{n}{3}\right\rceil p & \text { if } n & \equiv 1(\bmod 3) \\
\left.2\left\lfloor\frac{n}{3}\right\rfloor \mid \frac{p}{3}\right\rceil+\left(\left\lceil\frac{n}{3}\right\rceil+1\right) p & \text { if } n & \equiv 2(\bmod 3)
\end{aligned}\right.
$$

Proof is same as theorem 1.2.

Theorem 3.4 If $\mathrm{n} \leq \mathrm{p}$ and $\mathrm{p} \equiv 2(\bmod 3)$, then

$$
\gamma_{\text {rsd }}\left(\mathrm{C}_{\mathrm{p}} \otimes \mathrm{C}_{\mathrm{n}}\right)
$$

$$
\left\{\begin{array}{cc}
\left\lceil\frac{n}{3}\right\rceil\left(2\left\lceil\frac{p}{3}\right\rceil+p\right)+2 & \text { if } n \equiv 0(\bmod 3) \\
\left\lfloor\frac{n}{3}\right\rceil\left(2\left\lceil\frac{p}{3}\right\rceil\right)+2+\left\lceil\frac{n}{3}\right\rceil p & \text { if } n \equiv 1(\bmod 3) \\
\left\lfloor\frac{n}{3}\right\rfloor\left(2\left\lceil\frac{p}{3}\right\rceil\right)+2+\left(\left\lceil\frac{n}{3}\right\rceil+1\right) p & \text { if } n \equiv 2(\bmod 3)
\end{array}\right.
$$

Proof is same as theorem 1.2.

\section{CONCLUSION}

A conclusion section is not required. Although a conclusion may review the main points of the paper, do not replicate the abstract as the conclusion. A conclusion might elaborate on the importance of the work or suggest applications and extensions.

\section{ACKNOWLEDGMENT}

The research work was supported by DSA (Departmental special assistance) Gandhigram Rural Institute-Deemed to be university, Gandhigram under University Grants Commission- New Delhi .

\section{REFERENCES}

1. Gayla S. Domkea Johannes H. Hattingha Stephen T. Hedetniem Renu C. Laskar, Lisa R. Marku.(1999): Restrained domination in graphs, Discrete Mathematics, Vol. 203 pp. 61 - 69

2. Harary F, Graph Theory, Addison Wesley Reading Mass(1972).

3. Haynes T.W, S. T.Hedetniemi and P.J.Slater, Fundamentals of Domination in Graphs, Marcel Dekker, Inc., New York,1998

4. J.Paulraj Joseph, G.Mahadevan and A. Selvam, On complementary perfect domination number of a graph, Acta Ciencia Indica, (2006), 846-854.

5. Mahadevan .G, Iravithul Basira. A and M. Vimala Suganthi, Restrained step domination number of a graph, Advances in pure \& Applies Mathematics, ISBN 978-93-86435-54-5, pp 31-40.(2018)

6. Vizing, V.G., The cartesian product of graphs Vychisl. Sistemy, 9(1963)30-14

7. Mahadevan G and Ayisha B (2013): Further Results On complementary perfect domination number of a graph, International Mathematical Forum, Vol. 8, pp 85 -103.

8. J. Jagan Mohana, Indrani Kelkarb. (2012): Restrained 2-Domination Number of Compete Grid Graphs, International Journal of Applied Mathematics and Computation Journal, Vol 4(4), pp 352-358.

9. John Adrian Bondy, Murty U.S.R. (2008): Graph Theory, Springer

10. G.Mahadevan, A. Iravithul basira and C. Sivagnanam, "complementary connected perfect domination number of a graph", International Journal of Pure and applied Mathematics, Vol.106 No. 6 (2016), pp 17-24.

11. R. Kala and T. R. Nirmal Vasantha (2008): Restrained double domination number of a graph, AKCE J. Graphs. Combinatorics, Vol. 5, No.1, pp 73-82. 
12. Mahadevan G (2005): On domination theory and related concepts in graphs, Ph.D. thesis, Manonmaniam Sundaranar University, Tirunelveli, India.

\section{AUTHORS PROFILE}

Dr.G.Mahadevan M.Sc., M.Phil., M.Tech., Ph.D., is having 25 years of Teaching Experience in various Colleges and Universities, including Head of the department of Mathematics, at Anna University, Tirunelveli Region, Tirunelveli. Currently he is working as Asst.Professor, Dept.

of Mathematics, Gandhigram Rural Institute-Deemed to be University, Gandhigram. He is the Associate Editor of International Journal of Applied Graph theory. He reviewed many papers in reputed international/National Journals. He Published more than 90 research papers in various International/National Journals. He has produced Eight Ph.D's and many more students are pursuing Ph.D under his guidance. He has written three books on Engineering Mathematics and one book "Text book of Calculus". He Received Best Faculty Award-Senior Category in Mathematics, Mother Teresa Gold Medal Award, Dr.A.P.J Abdul Kalam Award for Scientific Excellence and Life Time Achievement Award for outstanding contribution in Education Field. He delivered more than 100 invited talks in various International/National Conferences. He served as Resource Person in various International Conferences at Asian Institute of Technology, Bangkok, Thailand, National University of Singapore, Singapore, Universiti Tunku Abdul Rahman, Malaysia, Build Bright University, Krong Siem Reap, Camboida et.

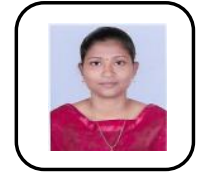

M. Vimala Suganthi, did her Post Graduation in Mathematics at Lady doak college, Madurai and did her M.Phil in Fatima College, Madurai. She has come out with flying colours by securing first class. She is currently doing research as Full Time Research Scholar under the guidance of Dr.G.Mahadevan, Dept. of Mathematics, Gandhigram Rural Insitute, Deemed to be University, Gandhigram. She presented many research articles in many international/National Conferences. 